\title{
Hidden Maxwell Stratum in Euler's Elastic Problem
}

\section{A. A. Ardentov}

This investigation continues the study of the classical problem of stationary configurations of an elastic rod on a plane. The length of the rod, the ends of the rod and the directions at the ends are fixed. The problem was first studied by Leonard Euler in 1744 and the optimal synthesis problem is still an open problem. Euler described a family of geodesics containing the solutions, which are called Euler elasticae. It is known that sufficiently small pieces of Euler elasticae are optimal, i.e., they have a minimum of the potential energy. In theory, the point where an optimal curve loses its optimality is called a cut point. Usually several optimal curves arrive at such points, so the points have multiplicity more than 1 and are called Maxwell points. The aim of this work is to describe numerically Maxwell points where two nonsymmetric elasticae come with the same length and energy value.

Keywords: Euler elastica, Maxwell strata, optimal control.

\section{Statement of the problem}

We consider the following optimal control problem [1]:

$$
\begin{aligned}
& q=(x, y, \theta) \in M=\mathbb{R}^{2} \times S^{1}, \quad u \in \mathbb{R}, \\
& \left\{\begin{array}{l}
\dot{x}=\cos \theta, \\
\dot{y}=\sin \theta, \\
\dot{\theta}=u,
\end{array}\right. \\
& q(0)=q_{0}=\left(x_{0}, y_{0}, \theta_{0}\right), \quad q(L)=q_{1}=\left(x_{1}, y_{1}, \theta_{1}\right), \\
& J=\frac{1}{2} \int_{0}^{T} u^{2} d t \rightarrow \min .
\end{aligned}
$$

Received May 04, 2019

Accepted October 03, 2019

This research was performed at the Ailamazyan Program Systems Institute of the Russian Academy of Sciences under the financial support from the Russian Science Foundation (project no. 17-11-01387).

Andrey A. Ardentov

aaa@pereslavl.ru

Ailamazyan Program Systems Institute of RAS

Pereslavl-Zalessky, Yaroslavl Region, 152020 Russia 
Here $u \in \mathbb{R}$ is unbounded control, it corresponds to curvature $\kappa$ of the trajectory $(x, y)$. The problem is known as the classical variational problem of stationary configurations of the elastic rod on a plane, which has been known for more than 275 years [2]. The minimization of the integral (1.4) corresponds to the minimization of the potential energy of the rod and the desired curve $(x, y)$ represents a profile of the rod with given boundary conditions (1.3) at the end points of the rod with the fixed length $L>0$.

System (1.2) is controllable with the natural condition $\left(x_{1}-x_{0}\right)^{2}+\left(y_{1}-y_{0}\right)^{2} \leqslant L^{2}$, moreover, equality is possible in the only case when $(x, y)$ is a straight line.

A possible solution of the problem (1.1)-(1.4) is a trajectory (geodesic) called Euler elastica. The family of all Euler elasticae was first described by Leonard Euler in 1744 [2] and later was named after him.

Euler elastica different from a straight line can be of two types: inflectional and noninflectional. Also, we distinguish two special subtypes of a noninflectional elastica: critical (with only one loop) and circle. It is known that small arcs of an elastica give a solution for the problem (1.1)-(1.4). However, large enough arcs are usually not optimal. The problem of describing all optimal arcs of elasticae is still an open problem. This problem is equivalent to that of finding the cut time along each elastica.

Definition 1. Cut time along a geodesic (elastica) is called the instant of time when the geodesic loses its optimality. The corresponding point on the geodesic is called the cut point. Cut locus is the set of all cut points.

\section{Known results}

Let us recall some known facts concerning the problem under consideration. One of the main reasons for an elastica to loose its optimality is a Maxwell point.

Definition 2. Maxwell point is a point where several different geodesics (in our case elasticae) with the same length $L$ and the same value of energy integral (1.4) meet one another. The corresponding instant of time is called Maxwell time. Maxwell points form Maxwell stratum (the term was introduced by V.I. Arnold in [3]).

After a Maxwell point (Maxwell time) a geodesic (elastica) can't be optimal. Usually a Maxwell point corresponds to some reflection symmetry, which provides the way for finding the cut time via the study of symmetries of the problem.

This work is based on the results obtained in a series of works $[1,4-6]$. There are three nontrivial discrete symmetries $\varepsilon^{1}, \varepsilon^{2}, \varepsilon^{3}$ for the problem, which generate corresponding Maxwell strata [1]. The known minimal Maxwell time

$$
\mathbf{t}(\lambda)=\min \left(t_{\mathrm{MAX}}^{1}(\lambda), t_{\mathrm{MAX}}^{2}(\lambda)\right)
$$

corresponding to the reflection symmetries provides an upper bound for the cut time $t_{\text {cut }}(\lambda) \leqslant \mathbf{t}(\lambda)$, where $\lambda$ is a covector which defines an elastica and $t_{\mathrm{MAX}}^{1}(\lambda), t_{\mathrm{MAX}}^{2}(\lambda)$ are Maxwell times corresponding to the symmetries $\varepsilon^{1}, \varepsilon^{2}$, respectively. A detailed description of the set of covectors $N \ni \lambda$ and an exponential mapping Exp: $N \rightarrow M$ which parametrizes elasticae is presented in [5]. The description of the global structure of the exponential mapping shows that there are Maxwell points which don't appear from known reflection symmetries $\varepsilon^{i}, i=1,2,3$. In fact, the upper bound $t_{\text {cut }}(\lambda) \leqslant \mathbf{t}(\lambda)$ is not sharp for some $\lambda$. The aim of this work is to describe numerically Maxwell points where two nonsymmetric elasticae come to with the same length and energy value. 


\section{Reduced problem}

Using the discrete symmetries $\varepsilon^{i}, i=1,2,3$, and the continuous symmetries (rotations, translations, dilations) it is possible to reduce the problem in the following way.

Lemma 1. Any optimal solution of the problem (1.1)-(1.3) can be transformed to the solution of the same problem (1.1)-(1.3) with the additional conditions

$$
\begin{aligned}
& T=1, \\
& x_{0}=y_{0}=0, \theta_{0}=-\theta_{1}, \\
& q_{1} \in \widehat{\mathcal{A}}=\left\{(x, y, \theta) \in M \mid x^{2}+y^{2}<1, y \geqslant 0, \theta \in[0, \pi / 2]\right\} .
\end{aligned}
$$

Remark 1. We exclude the case of a straight line for simplicity. Further, we assume that conditions (3.1)-(3.3) hold for the problem under consideration.

Let us state several lemmas concerning the problem (1.1)-(3.3).

Lemma 2. The control problem (1.1)-(1.3), (3.1)-(3.3) has continuum solutions.

Lemma 3. There are a countable number of noninflectional elasticae which give the solution of the problem (1.1)-(1.3), (3.1)-(3.3). All such solutions are locally minimizing functional (1.4).

Solutions of the problem (1.1)-(3.3) for the cases

$$
\begin{aligned}
& q_{1} \in M_{\theta}=\{q \in \widehat{\mathcal{A}} \mid \theta=0\}, \\
& q_{1} \in M_{P}=\{q \in \widehat{\mathcal{A}} \mid y=0\}
\end{aligned}
$$

were investigated in [6].

We prove the following statements.

Theorem 1. There are not more than four optimal elasticae which provide a solution to the problem (1.1)-(3.3).

Theorem 2. If the problem (1.1)-(3.3) has three or four optimal solutions, then condition (3.4) holds.

Corollary 1. There exists not more than two inflectional and not more than two noninflectional elasticae which provide a solution to the problem (1.1)-(3.3).

\section{Hidden Maxwell stratum}

Corollary 2. The problem (1.1)-(3.3) with the condition

$$
q_{1} \in \widehat{\mathcal{A} \backslash M_{\theta}}
$$

has one or two optimal solutions.

Definition 3. Denote by $\mathcal{H}$ a set of points where two optimal solutions of the problem (1.1)-(3.3) come with condition (4.1). We call the set $\mathcal{H}$ hidden Maxwell stratum.

Proposition 1. If $q_{1} \in \overline{\mathcal{H}} \cap M_{\theta}$, then the problem (1.1)-(3.3) has three or four optimal solutions.

Figure 1 shows sections of the cut locus Cut formed by the intersection with the planes $\left\{y_{1}=0\right\},\left\{\theta_{1}=0\right\},\left\{\theta_{1}=\pi / 2\right\}$ in the domain $\widehat{\mathcal{A}}$. The construction of those sections is given in $[6]$. 


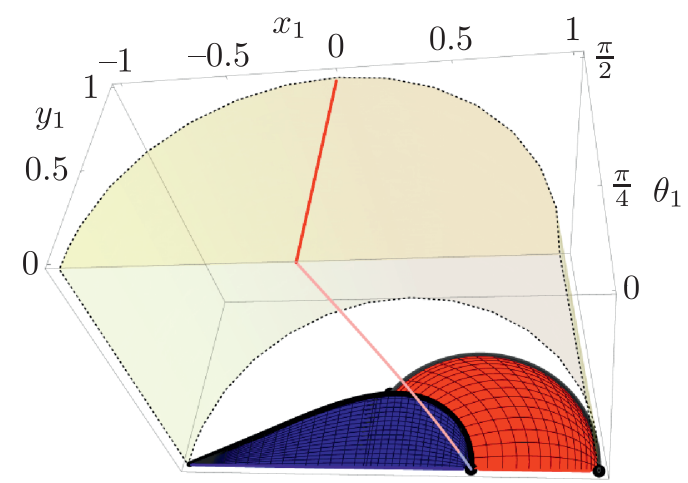

Fig. 1. Sections of the cut locus formed by the intersection with the planes $\left\{y_{1}=0\right\},\left\{\theta_{1}=0\right\},\left\{\theta_{1}=\pi / 2\right\}$.

Proposition 2. Cut locus Cut $\subset \widehat{\mathcal{A}}$ for the problem (1.1)-(3.3) is contained in the union $\mathcal{H} \cup M_{\theta}$.

Proposition 3. The following equality holds:

$$
\text { Cut } \cap\{\theta=\pi / 2\}=\{(x, y, \theta) \in \widehat{\mathcal{A}} \mid x=0, \theta=\pi / 2\}=M_{Q} .
$$

REMARK 2. The set $M_{Q}$ represents the Maxwell points corresponding to symmetry $\varepsilon^{3}$ which doesn't play an important role in constructing the upper bound $\mathbf{t}(\lambda)$ for the cut time obtained in [1]. However, it turns out that these points belong to the defined hidden Maxwell stratum $M_{Q}=\mathcal{H} \cap\{q \in \widehat{\mathcal{A}} \mid \theta=\pi / 2\}$.

We construct the hidden Maxwell stratum $\mathcal{H}$ inside $\widehat{\mathcal{A}}$ by solving the following system of equations:

$$
\left\{\begin{array}{l}
\operatorname{Exp}\left(\lambda_{1}\right)=\operatorname{Exp}\left(\lambda_{2}\right) \\
J\left(\lambda_{1}\right)=J\left(\lambda_{2}\right)
\end{array}\right.
$$

where Exp is the exponential mapping, which translates a covector $\lambda$ to the end point of the corresponding elastica with length $1 ; \lambda_{1}, \lambda_{2}$ are covectors for elasticae (for short we will not give the definitions of the domains for those parameters); $J(\lambda)$ is the value of integral (1.4) for an elastica with length 1 corresponding to a covector $\lambda$.

Notice that system (4.3) is a four-dimensional system given by the Jacobi elliptic functions and the elliptic integrals of the first and second kind. It seems impossible to resolve such a system analytically. In order to solve system (4.3) efficiently, we derive simplified formulas for the exponential mapping Exp for each type of elastica. Then we develop a program for a numerical solution of system (4.3) in computing system Wolfram Mathematica.

The numerical investigation shows that one of the covectors $\lambda_{1}, \lambda_{2}$ corresponds to an inflectional elastica, otherwise system (4.3) doesn't have a solution, so we associate $\lambda_{1}$ with an inflectional elastica, $\lambda_{2}$ is associated with an inflectional or noninflectional (including critical) elastica.

First, we study solutions when $\lambda_{2}$ corresponds to a critical elastica with $\mathbf{t}\left(\lambda_{2}\right)=\infty$, i.e., there is no bound on the cut time for a critical elastica. However, the numerical examination shows that there are nonoptimal pieces of such an elastica. Figure 2 shows a pair of critical and inflectional elasticae arriving at the point

$$
x_{1} \approx 0.38338, \quad y_{1}=0, \quad \theta_{1} \approx 0.15761 .
$$




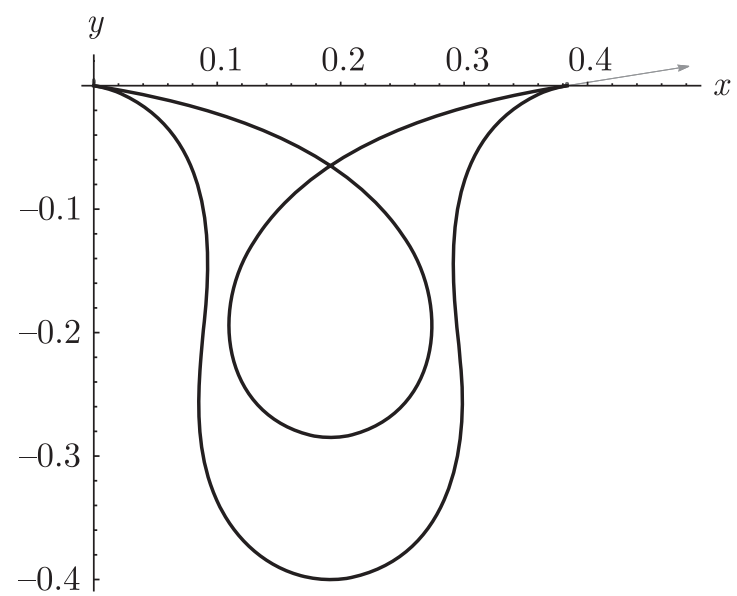

Fig. 2. An example of critical and inflectional elasticae arriving at the hidden Maxwell strata.

Starting from this point we construct solutions of such type by continuity, see the dashed line in Fig. 3.

Further, we construct other types of solutions by continuity. A current picture of the cut locus is presented in Fig. 4. The points over the dashed line represent the case when $\lambda_{2}$ corresponds to an inflectional elastica and the points under the dashed line represent the case when $\lambda_{2}$ corresponds to a noninflectional elastica. We found around half million solutions of system (4.3) to obtain this picture.

We see that the case when $\lambda_{2}$ corresponds to an inflectional elastica is almost complete. However, the noninflectional case is just half filled. During calculation we face an obstacle to calculate solutions close to the cylinder $x_{1}^{2}+y_{1}^{2}=1$, since the value of the energy integral near such points tends to infinity.

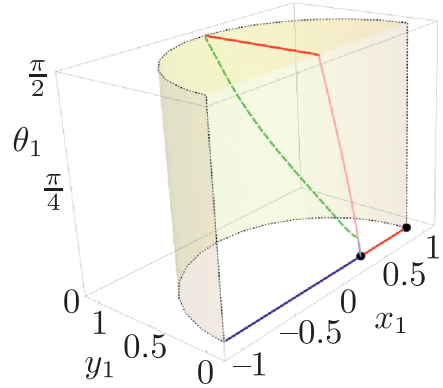

Fig. 3. The dashed line is part of the Hidden Maxwell strata corresponding to critical elastica.

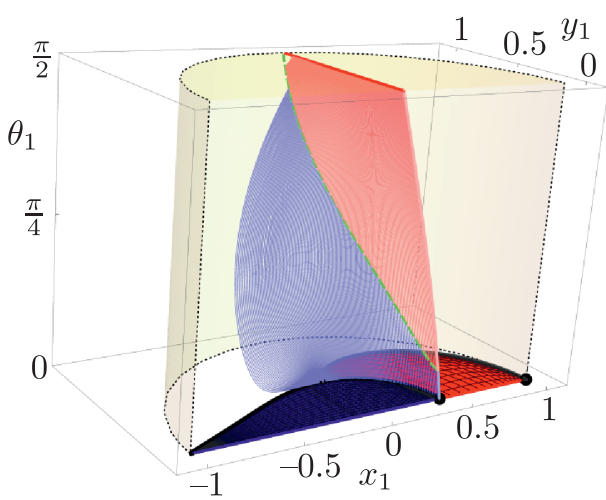

Fig. 4. The current picture of the cut locus.

Our further aim is to describe numerically the hidden Maxwell set near the cylinder $x_{1}^{2}+y_{1}^{2}=1$ and propose an analytic description of its limit on the cylinder. At present, we know only two limit points: $x_{1}=-1, y_{1}=\theta_{1}=0$ and $x_{1}=0, y_{1}=1, \theta_{1}=\pi / 2$. 


\section{References}

[1] Sachkov, Yu. L., Maxwell Strata in Euler's Elastic Problem, J. Dyn. Contr. Syst., 2008, vol. 14, no. 2, pp. 169-234.

[2] Euler, L., Methodus inveniendi lineas curvas maximi minimive proprietate gaudentes, sive solutio problematis isoperimetrici lattissimo sensu accepti: Additamentum 1. De curvis elasticis, Lausanne: Bousquet, 1744, pp. 245-310.

[3] Arnold, V. I., Gusein-Zade, S. M., and Varchenko, A. N., Singularities of Differentiable Maps: Vol. 1. Classification of Critical Points, Caustics and Wave Fronts, Modern Birkhäuser Classics, New York: Birkhäuser/Springer, 2012.

Arnold, V.I., Gusein-Zade, S.M., and Varchenko, A.N., Singularities of Differentiable Maps: Vol.2. Monodromy and Asymptotics of Integrals, Modern Birkhäuser Classics, New York: Birkhäuser/Springer, 2012.

[4] Sachkov, Yu. L., Conjugate Points in Euler's Elastic Problem, J. Dyn. Control Syst., 2008, vol. 14, no. 3, pp. 409-439.

[5] Sachkov, Yu. L. and Sachkova, E. L., Exponential Mapping in Euler's Elastic Problem, J. Dyn. Control Syst., 2014, vol. 20, no. 4, pp.443-464.

[6] Ardentov, A. A., Multiple Solutions in Euler's Elastic Problem, Autom. Remote Control, 2018, vol. 79, no. 7, pp. 1191-1206; see also: Avtomat. $i$ Telemekh., 2018, no. 7, pp. 22-40. 